and yet the blood picture does not help to confirm such a classification. Considering the question of lympho-sarcoma, it is true that such a growth may remain encapsulated for a considerable time, even for a year, but in this case there does not appear to have been any great increase in size of the growth and no evidence of any metastases. The glands, etc., in the abdomen found post-mortem would be in keeping, however, with the leukaemic theory. Further, H. P. L. Wells and M. S. Mayou, Trans. Opthal. Soc. U.K., XXX, (1910), p. 97, point out that lympho-sarcomata of the lacrymal gland are usually met with in persons over 38 years of age.

It is, therefore, our belief that in this case the growth in the lacrymal gland was a leukaemic infiltration.

\title{
THE PHOTOCHROMATIC INTERVAL IN GLAUCOMA AND CAVERNOUS ATROPHY
}

BY

RANSOM PICKARD

EXETER

IN searching for some test which might prove useful in eyes which were possibly glaucomatous, a study was made of the field photochromatic interval. The results in glaucoma and cavernous atrophy seem worth communicating, in the hope that this test may be tried on a larger scale, further to test its usefulness.

In this paper the photochromatic interval will be abbreviated into "p.c.i." It is the condition in which a colour produces stimulus of light but not of colour. It can be produced in two ways; at a fixed spot by a minimal stimulus, which can be converted into a colour sensation by sufficient increase in brightness or enlargement of the area stimulated; or by movement in the field from without inwards to the fixation point. The area through which the colour stimulus is perceived as light, not colour, is the p.c.i. In this paper only the field is dealt with, and a moving object employed.

In the tests full daylight, varying from 150 to 240 foot candles at one metre, was employed with a Bjerrum screen as a background. "Ilford" coloured gelatin, mounted on pot opal, masked down to the various required sizes, was used. The opal was fastened to a flat piece of metal, from one end of which a bicycle brake wire, two inches long, was held on a Traquair's rod. By this means transmitted colours were used, not reflected.

Some preliminary experiments were made by the writer on his 
right eye, to give data for comparison. They were made with the following colours :-

$$
\begin{aligned}
& \text { Ilford Tricolour-Green. } \\
& \text { Ilford Tricolour-Red. } \\
& \text { Ilford Micro 4 (Yellow). } \\
& \text { Ilford Minus Red (Blue). }
\end{aligned}
$$

As used, in daylight, with the pot opal background, it is obvious that the daylight passed through the gelatines twice. By courtesy of the Ilford Company, a chart of the transmission

\section{ChART A}

Transmission data of some Ilford Gelatin Filters.

The transmission curves of the single filters are shown by dotted lines.

The transmission curves for the doubled filters are show by full lines.

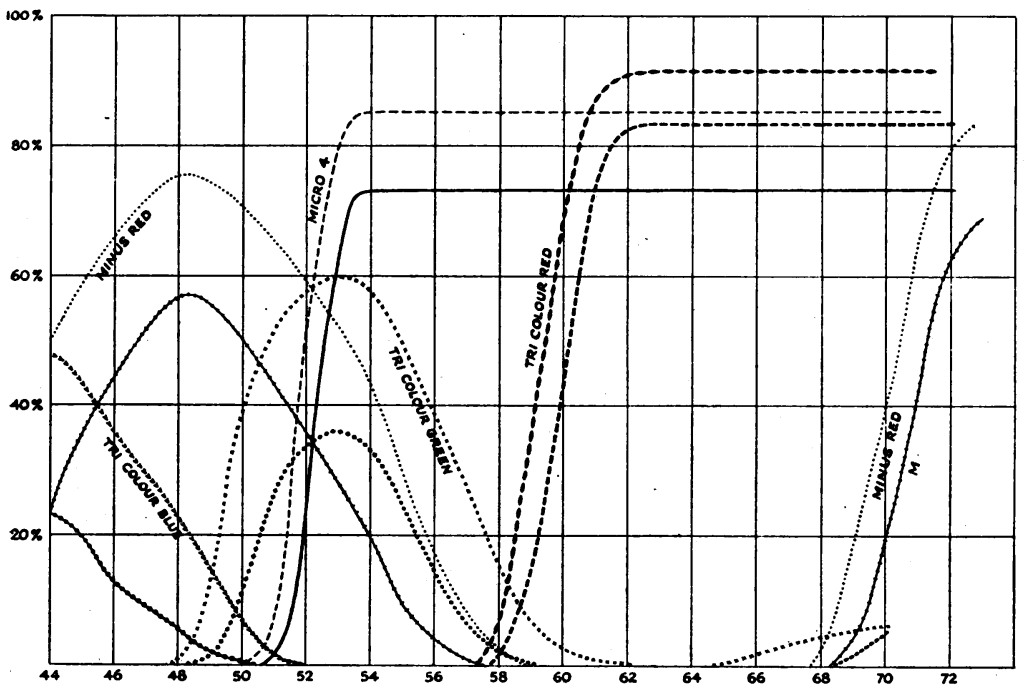

\begin{tabular}{|c|c|c|c|c|}
\hline & & & Spectrum included & Excluded \\
\hline Green & - & $\begin{array}{l}\text { Per cent. } \\
\quad 36\end{array}$ & Green & Red, yellow \\
\hline Red & - & 83 & Red & $\begin{array}{l}\text { Blue, green, part } \\
\text { yellow }\end{array}$ \\
\hline Yellow & - & 63 & Yellow, red & Blue, green \\
\hline Blue & - & 57 & Blue, part green & Yellow and red \\
\hline
\end{tabular}

By permission of the Ilford Company, Ltd.

values for the four colours is shown. The double transmission colour values are :- 
The fields for circular discs varying from 1 to $10 \mathrm{~mm}$. in diameter, the colours as above, were used to ascertain the normal photochromatic interval. The results are summarised in four charts $(b-e)$. The average radii were ascertained by adding together the eight observations and dividing by eight. The abscissae represent the angles from the fixation point thus obtained. The co-ordinates are arbitrary values, representing the inverse of the diameters of the coloured discs multiplied by 10 . Thus $1 \mathrm{~mm} .=10,1 / 10 \mathrm{~mm} . \times 10=1$, representing the sensitivities according to Weber's law.

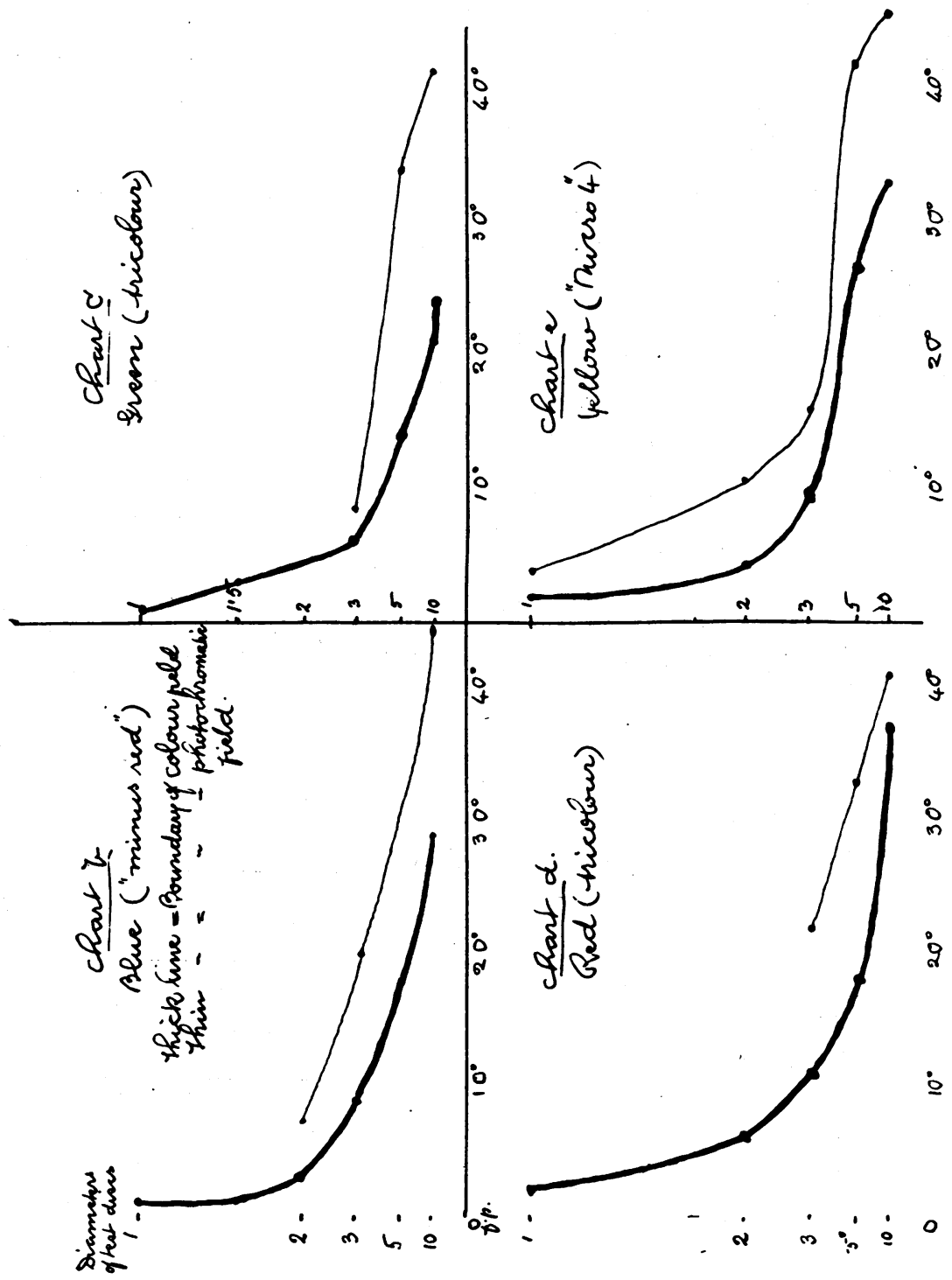


It will be seen that, while the photochromatic interval persists with the yellow discs as far as the $1 \mathrm{~mm}$. disc, it is not so with the other colours. With the blue it ceases at the $2 \mathrm{~mm}$. disc, with green and red at the $3 \mathrm{~mm}$. disc. A comparison with the transmitted spectral values shows that the interval does not vary with these; for with the red having a transmission of 80 per cent. and the green of 36 per cent. the interval stops with the $3 \mathrm{~mm}$. disc.

A brightness test in electric light, the filters being mounted on pot opal, thus giving double transmission as above, gave these results :-

$\begin{array}{llllr}\text { Pot opal } & \ldots & \ldots & \ldots & 100 \text { per cent. } \\ \text { Yellow } & \ldots & \ldots & \ldots & 100 \text { per cent. } \\ \text { Red } & \ldots & \ldots & \ldots & 46 \text { per cent. } \\ \text { Blue } & \ldots & \ldots & \ldots & 38.5 \text { per cent. } \\ \text { Green } & \ldots & \ldots & \ldots & 33 \text { per cent. }\end{array}$

It will thus be seen that the comparisons are not accurate because the brightness values differ. Even so, the photochromatic interva! persistence for the smaller objects does not follow the brightness values, except with yellow.

In investigating this subject clinically it was considered better to use the red only, because the change from white to red is more easily recognised by an untrained person than of white to yellow or blue. Only the $10 \mathrm{~mm}$. disc was used in the group of cases dealt with in this paper. This has the advantage of making the issue more clear cut and the cases more comparable with each other. Generally speaking the transition from white to red in moving the objects from the periphery to the centre of the field is fairly sudden; but with some it is gradual, the intermediate zone being called pink, with yet others-and more rarely, it is called yellow.

If the rise into consciousness as red be represented by a slope, the sudden onset of red may be represented as a sudden increase of the slope A, the other as a steady rise, B.

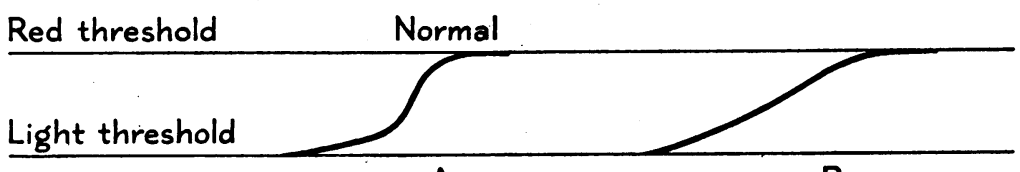

A

B

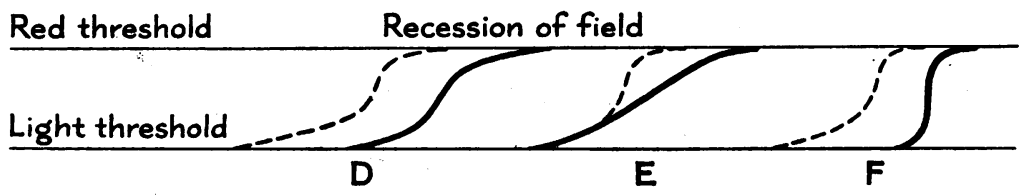


On a priori grounds there are three obvious ways by which the photochromatic interval may be altered. Taking $\mathrm{A}$ as the commoner normal condition, $D$ represents an equal diminution of perception for the p.c.i. interval and the red, $E$ a diminution of the red field and increase of the p.c.i. interval, $F$ a complete loss of the p.c.i. and slight alteration in the red field. Obviously these differences can be combined in various degrees, showing themselves as decreases or increases in the fields, either of the p.c.i. or red.

Opportunity has not offered itself to investigate the p.c.i. in sufficient cases of nervous or cerebral disease to make any report on them now, it is proposed to deal only with groups of glaucoma and cavernous atrophy at present.

The cases taken are consecutive and not selected, they are random in the proper sense because they came for examination without arrangement or collusion. It so happens that eighteen of each class occurred in the period of investigation. Dividing each group into two classes, those with a p.c.i. of under $5^{\circ}$, or $5^{\circ}$ and over, the numbers are these :-

\begin{tabular}{|c|c|c|c|}
\hline p.c.i. & & Glaucoma & Cavernous atrophy \\
\hline Under $5^{\circ}$ & 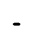 & 15 & 4 \\
\hline $5^{\circ}$ or over & - & 3 & 14 \\
\hline
\end{tabular}

The question at once arises whether this curious reversal in the classes in the two groups is mere chance, if another set of cases would have shown quite a different class grouping. This is possible but most improbable as the following arguments will show. The grouping of the p.c.i. into two classes is an example similar to coin tossing or any other two alternatives. In dealing with eighteen chances the possibilities are shown by the development of $(1+1)^{18}$, according to the arithmetical triangle of Jevons. Thus in grouping $3 / 15$ the chances are $816 / 262,144$ or 1 in 321 ; in $4 / 14$ they are $3,060 / 262,144$ or 1 in 85 .

To supplement this method an actual experiment was made with coloured beads of equal size and spherical shape, amethyst and yellow, an equal number of each (41). These were mixed in a bag, a handful taken out and a bead dropped alternately in each of two cups till eighteen beads were in each. The number of each colour in each cup was then counted. In 162 counts the actual distribution was :- 
TABLE I

Probability Chart

\begin{tabular}{|c|c|c|c|}
\hline Amethyst & Yellow & $\begin{array}{c}\text { Actual } \\
\text { Grouping } \\
(162 \text { counts })\end{array}$ & $\begin{array}{c}\text { Theoretical } \\
\text { Distribution } \\
(1+1)^{18}\end{array}$ \\
\hline 0 & 18 & .- & 1 \\
\hline 1 & 17 & - & 18 \\
\hline 2 & 16 & - & 153 \\
\hline 3 & 15 & 1 & 816 \\
\hline 4 & 14 & 1 & 3060 \\
\hline 5 & 13 & 12 & 8568 \\
\hline 6 & 12 & 18 & 18564 \\
\hline 7 & 11 & 23 & 31824 \\
\hline 8 & 10 & 35 & 43758 \\
\hline 9 & 9 & 33 & 48620 \\
\hline 10 & 8 & 16 & 43758 \\
\hline 11 & 7 & 8 & 31824 \\
\hline 12 & 6 & 2 & 18564 \\
\hline 13 & 5 & 4 & 8568 \\
\hline 14 & 4 & - & 3060 \\
\hline 15 & 3 & 1 & 816 \\
\hline 16 & 2 & - & 153 \\
\hline 17 & 1 & - & 18 \\
\hline 0 & 18 & - & 1 \\
\hline
\end{tabular}

Giving 1 count of the A15/Y3 group and 1 of the A3/Y15 group in 162 trials, 1 and 0 in the $14 / 4$ groups. But the proba. bility is much less than this. For the coincidence of the 15 amethyst 3 yellow with the 4 amethyst 14 yellow would be far less probable than the occurrence of either group with some other combination. Thus the two groups considered together are so improbable as a matter of chance that this may be neglected. They may be dealt with as due to some cause or causes other than chance.

In the chart " $f$ " and Table II the cases are arranged in order of size of the red field, as found by a $10 \mathrm{~mm}$. disc of Ilford Tricolour red mounted on pot opal. It will be seen that of the glaucoma cases, 12 are $14^{\circ}$ or less, 6 being spread out between 


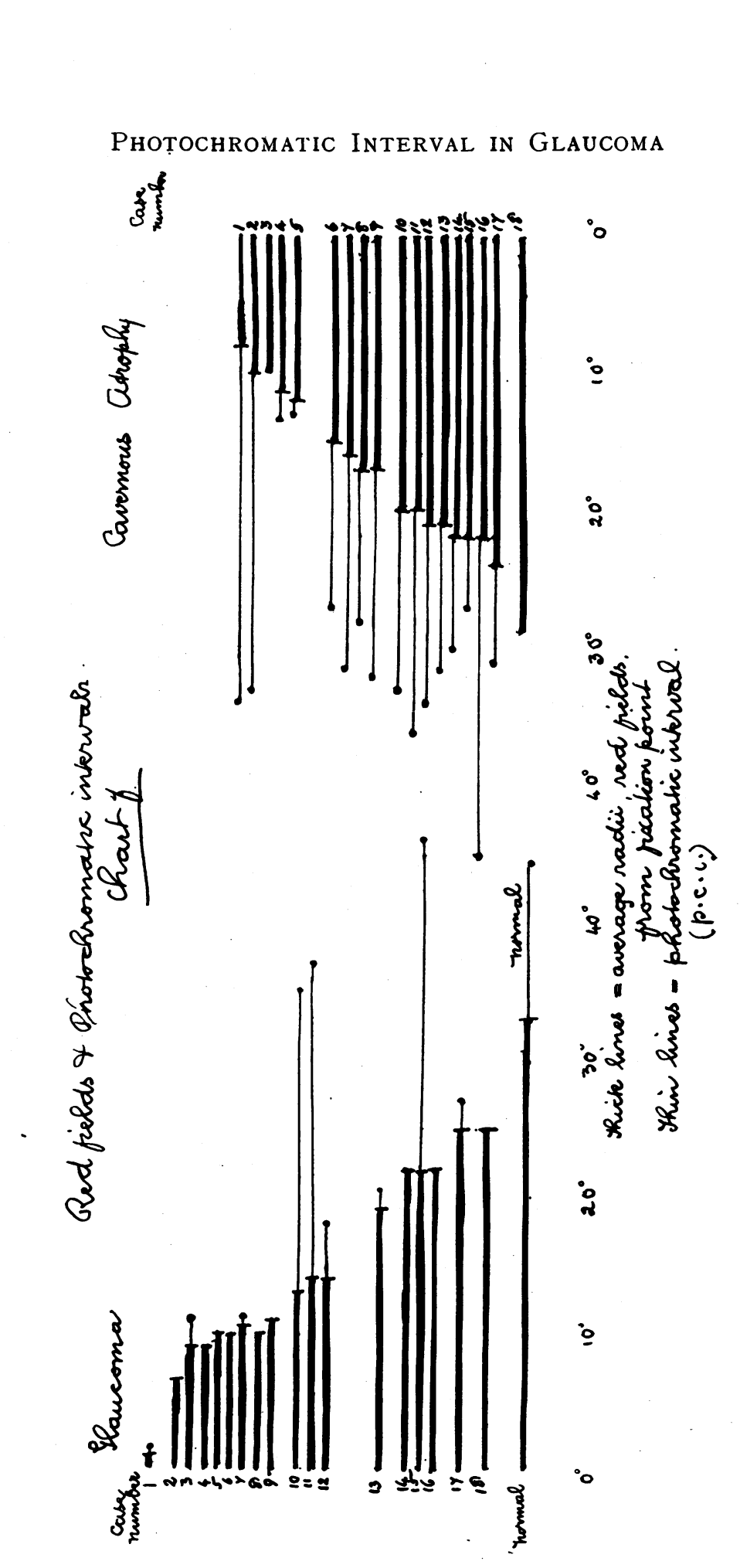

397

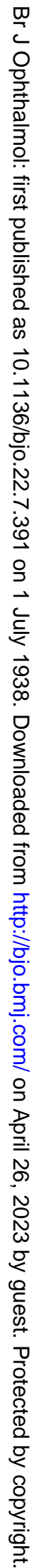




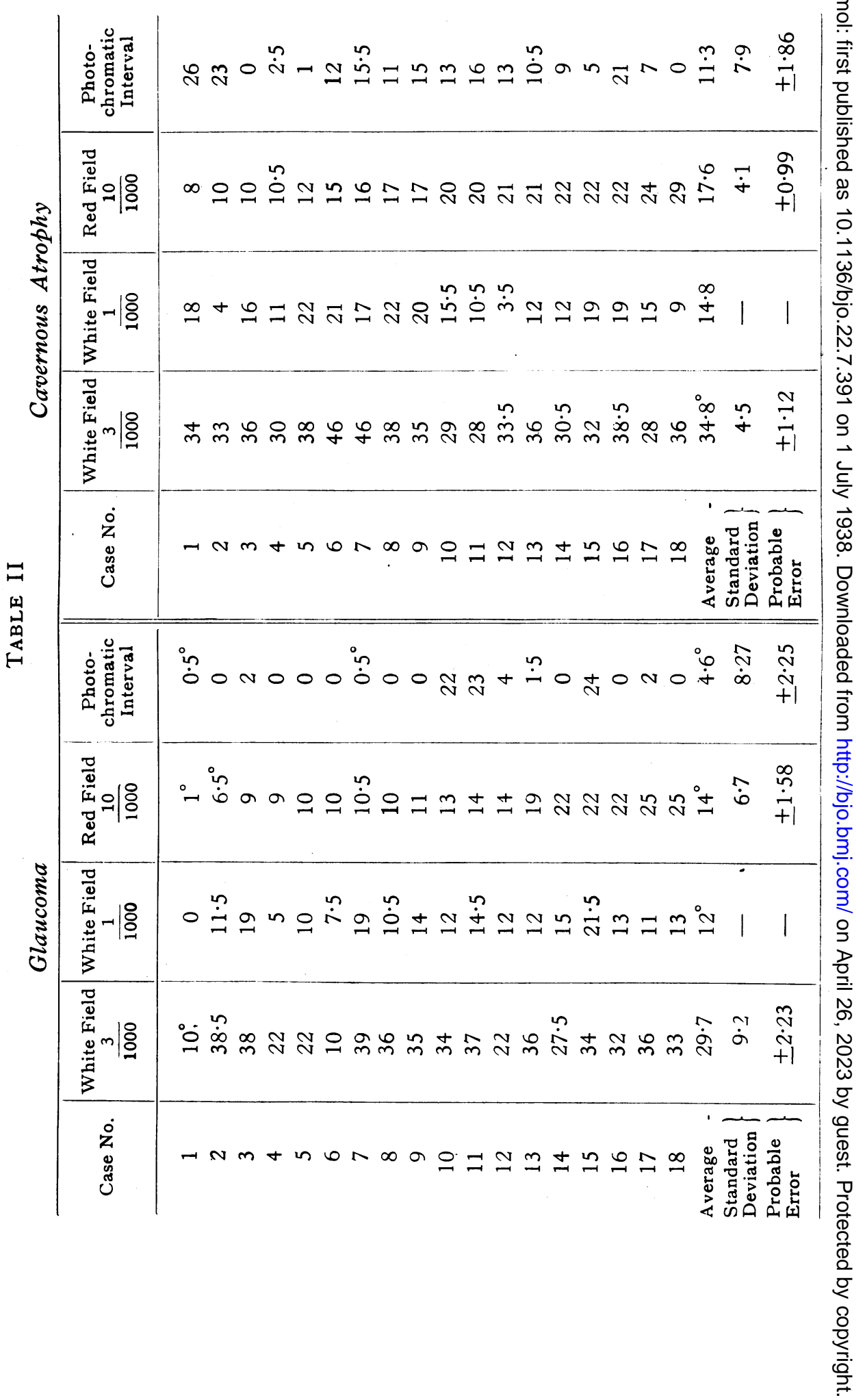


$19^{\circ}$ and $25^{\circ}$. The cavernous atrophy group shows 6 cases of $15^{\circ}$ or less, 11 between $16^{\circ}$ and $26^{\circ}$, and 1 of $28^{\circ}$. But the marked feature of the photochromatic interval in the glaucoma group is that it is absent or slight in every case but three, whereas it is present and large in all the cavernous atrophy cases except four, and in one of these four the red field is normal; if allowance is made for the variation in the normal red field that actually occurs.

Moreover, the chart shows that, if the red field and the p.c.i. be added together, the total field in the cavernous atrophy cases is not far short of the normal. In other words, these cases are examples of the " $E$ " diagram method of decrease of the red field, the outer boundary of the p.c.i. being less affected than the red boundary. It will be seen that with the three exceptions in one group and four in the other, this distinction occurs throughout the whole series, irrespective of the size of the red field, an important point. The groups are in this respect curiously the converse of one another. Although this distinction is not absolute, there being the exceptions named in each group, it is sufficiently frequent to be a notable difference. That there are exceptions makes it more difficult to apply this rule to any particular case; but it may fairly be said that, if the red field is diminished and the photochromatic interval is under $5^{\circ}$, there is a five to one chance that it is glaucoma. Conversely, if the red is reduced and the p.c.i. is over $5^{\circ}$, the chances are one to four that it is cavernous atrophy.

The question as to whether the two groups, glaucoma and cavernous atrophy, are of comparable severity is important. As $3 / 1,000$ white and $10 / 1,000$ red give nearly the same field, if the p.c.i. be excluded, it is proposed to use the $3 / 1,000$ white field as a standard of comparison. The full details are given in Table II.

It will be seen that the glaucoma $3 / 1,000$ white field gives an average of $29.7^{\circ}$, cavernous atrophy of $34.8^{\circ}$, a difference of $5 \cdot 1^{\circ}$, not great but decided. Thus on the whole the glaucoma series is more severe than the cavernous atrophy group. Yet it will be seen on inspection of the chart that the small or absent p.c.i. is well scattered over the whole of the glaucoma group, it is not confined to the severe cases. In the cavernous atrophy group the absent p.c.i. occurs in those with nearly average $3 / 1,000$ white field.

The average of the red fields shows a difference of $3.6^{\circ}$ between the two groups, which is almost within the range of patients' errors. But the p.c.i. is $4.6^{\circ}$ in the glaucoma cases as against $11.3^{\circ}$ in the cavernous atrophy group, a very marked distinction.

In clinical work we are often confronted with the problem 
whether an eye is affected by glaucoma or cavernous atrophy, or whether it is healthy. Tension, white fields and central vision may be normal when examined. This question becomes more urgent when one eye has, without question, glaucoma, possibly in an advanced stage. Such a case was No. 9 in the glaucoma series; but the red field was down to $11^{\circ}$, with no p.c.i. There was additional confirmation of affection of this eye in a slightly lowered central light threshold ( $0001875 \mathrm{ft}$. candles) and in the presence of two white fragments adherent to the pupil margin. The other eye had advanced glaucoma lento-exfoliativa.

It is easier to state the facts than to give an explanation of these alterations in the photochromatic interval. As the clinical defects do not go further back than the optic nerve, the explanation must allot the cause between the retina and the conducting fibres. In glaucoma the retinal visual elements are directly affected by pressure and so are the fibres, in the retina and the optic nerve head. In cavernous atrophy there is no pressure on the retina, whereas the nerve fibres are involved in the contraction of the nerve head. It would seem that pressure on the retina extinguishes the p.c.i. portion of the red field, as though that part of the retina were more susceptible to pressure than the area where red is seen as colour. Now the latter corresponds fairly closely to the $3 / 1,000$ white field, which is found clinically to stand pressure in glaucoma better than the $1 / 1,000$ or the $5 / 300$ white field. But this explanation would be valid only if the red field remained of normal size, which is not the case in the glaucoma series. Therefore, the more general explanation must be adopted that pressure quickly obliterates the liminal subchromatic red sensation in whichever part of the retina this may be. The exceptional three glaucoma cases must be taken as indicating the possibility of occasional great powers of resistance.

Of the four exceptions in the cavernous atrophy group, one has a practically normal colour field. The obvious explanation of the other three is that they were near the $10^{\circ}$ red field limit, within which the p.c.i. soon disappears. Yet it will be seen that cases 1 and 2 in this series have a large p.c.i. although the red fields are $8^{\circ}$ and $10^{\circ}$ respectively.

It would seem that more experience is required with this test, in cases where there is known to be nerve interruption, before any adequate explanation can be given.

In conclusion, this test of the size or absence of the photochromatic interval, is put forward as worthy of further investigation, in the hope that it may be of use, particularly in the early stages of glaucoma. 